\title{
Line-Structure Laser Human Body 3D Scanner with High Resolution
}

\author{
Tian QINGGUO*1,2, Zhang XIANGYU ${ }^{1,2}$, Li YUNPENG ${ }^{1,2}$, Yang YUJIE ${ }^{1,2}$, \\ Wang JINJIANG ${ }^{1,2}$, Ge BAOZHEN ${ }^{1,2}$ \\ ${ }^{1}$ School of Precision Instrument and Opto-Electronics Engineering, Tianjin University, Tianjin, China; \\ ${ }^{2}$ Key Laboratory of Opto-Electronics Information Technology of Ministry of Education, Tianjin, China
}

http://dx.doi.org/10.15221/13.195

\begin{abstract}
At present, digital human modeling (DHM) is a research hotspot due to it has wide applications. This paper develops a high resolution human body 3D scanner which conforms to triangulation principle and adopts line-structure light scanning mode. In accordance with modular design concept, the scanner can be divided into optical sensing and movement mechanism module, motion control and image capturing module, image processing and 3D reconstruction module. The scanner is composed of 4 pillars which create a square with the diagonal length $1644 \mathrm{~mm}$ between optical sensors, and the pillar's height is $3050 \mathrm{~mm}$. the measurement volume is a cylinder with radius (in horizontal plane) $\mathrm{R}=500 \mathrm{~mm}$ and height (vertical orientation) $\mathrm{Hv}=2000 \mathrm{~mm}$. there are 8 optical sensors totally in the scanner, and the typical resolution of each optical sensor is $0.7 \mathrm{~mm} / \mathrm{pixel}$ along $\mathrm{x}$ axis and $1.0 \mathrm{~mm} / \mathrm{pixel}$ along y axis. The scanning resolution can be changed from $2 \mathrm{~mm}$ to $4 \mathrm{~mm}$, and the time used is from 20 s to 10 s correspondingly. The reprojection mean error displays calibration results is better than $0.35 \mathrm{~mm}$ using a dynamic target designed with $2 \mathrm{D}$ electric translation stages and ceramic gauge block. The point cloud acquired from a real person shows that the scanner is high effective though some aspects should be improved later.
\end{abstract}

Keywords: Digital Human Modeling; 3D scanner; triangulation principle; line-structure light scanning; modular design

\section{Introduction}

Digital Human Modeling (DHM), a research hotspot for many scientists in recent years, owns a wide range of applications, such as costume design automation and virtual try-on [1 3], Ergonomic Design [4]、Medicine and Rehabilitation Engineering [5], Motion capture technology [6,7] and the field of artificial intelligence and cognitive intelligence model. So it has become increasingly important to make the study of quick and accurate human body 3D digital measurement technology.

Because of some characteristics such as non-contact, high level of speed, automation and accuracy, optical methods are suitable for human body surface 3D digital modeling. Optical methods can be divided into passive and active optical methods. Passive optical measurement does not require any auxiliary light source by using the surface reflected light, such as passive stereo vision [8]; active optical methods need to project the structured light on the surface of the measured object, then by receiving the modulated scattered light to achieve 3D measurements, such as the grating pattern projection method [9], laser triangulation method [10, 11], flight time method.

Laser triangulation method uses similar triangles between the objects and the graphics to achieve the depth information of object. Compared with other active measurement methods, this method is easy to handle and control with a simple design. According to the structural characteristics of the light source, the technology can be divided into point-structure light scanning [12], line-structure light scanning [13], multiple line-structure light scanning [14], and so on.

Based on the laser triangulation principle, this article adopts line-structure light scanning and multi-sensor simultaneous measurement technology to develop a high-resolution 3D laser human body scanner. With the modular design concept, this scanner is divided into optical sensing and movement mechanism module, motion control and image capturing module, image processing and 3D reconstruction module. After calibrated by precision calibration target designed with high-precision $2 \mathrm{D}$ electric translation stage and ceramic gauge block, the scanner can acquire 3D point cloud of human body profile quickly and accurately.

\footnotetext{
* tianqingguo@tju.edu.cn
} 


\section{Human body 3D scanner's general structure and module design}

The human body 3D scanner designed here is composed of several modules and its general structure diagram is shown in Fig. 1. Under the fully consideration of human body size statistics, the maximum measurement volume is a cylinder of radius (in horizontal plane) $R=500 \mathrm{~mm}$ by height (vertical orientation) $H_{v}=2000 \mathrm{~mm}$. The designed horizontal resolution $r_{h}$ (x axis orientation in Fig. 2(b)) and vertical resolution $r_{v}$ (y axis orientation in Fig. $2(b)$ ) are $0.7 \mathrm{~mm} /$ pixel and $1.0 \mathrm{~mm} /$ pixel respectively. Four pillars make a square and human body under test stands on a platform at its center. On each pillar the optical sensor and mechanism module is installed which can move smoothly up and down controlled by motion control and image capturing module to scan human profile and capture light stripe image. Light stripe image process module calculates light stripe's sub-pixel level centerlines and then calculates 3D profile point's coordinates taking advantage of system calibration results.

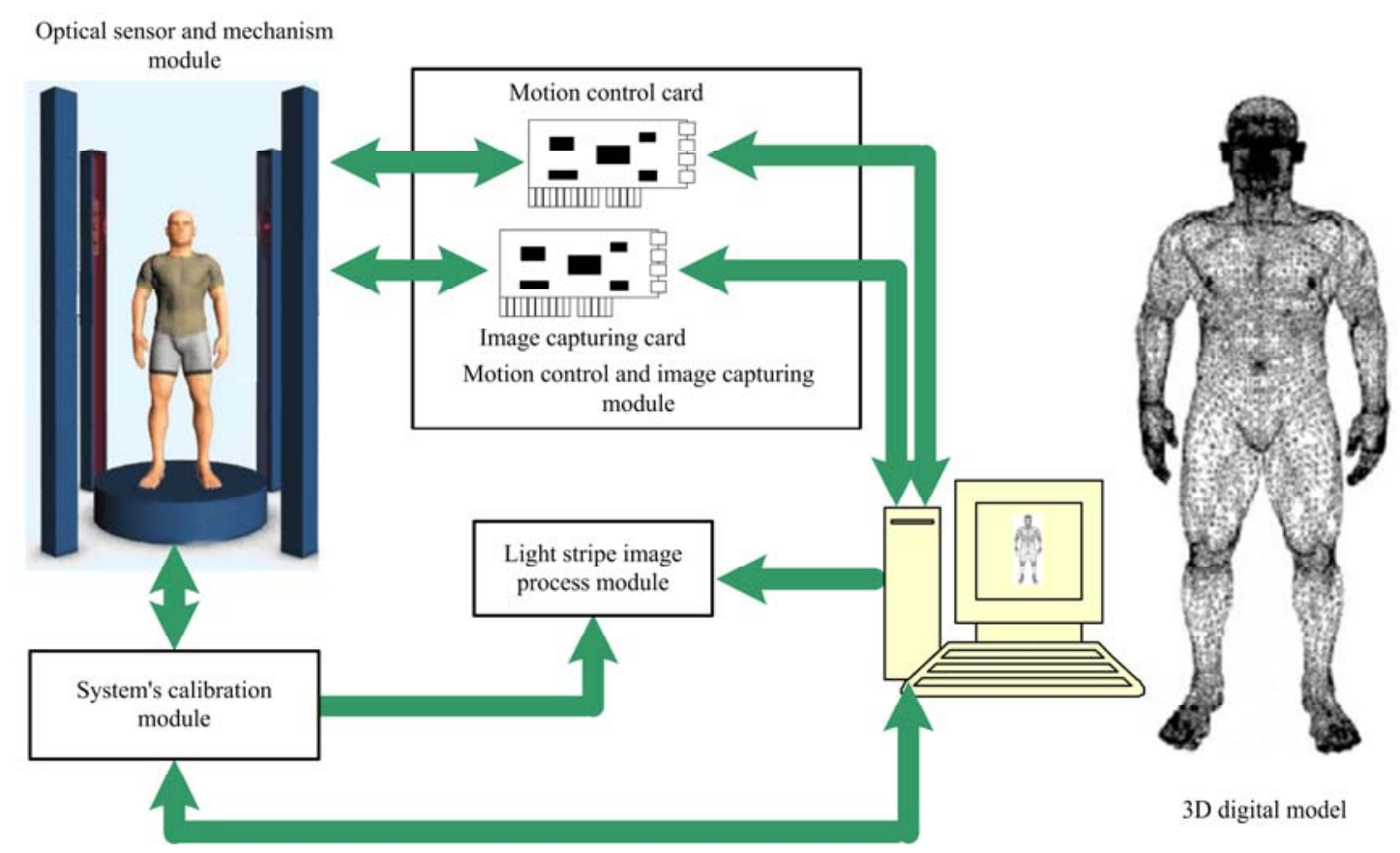

Fig. 1 The general structure diagram of human body 3D scanner.

\subsection{Optical sensing and movement mechanism module}

\subsubsection{Optical design}

Optical path is based on triangulation theory, and light source is selected as line-structure laser source. The top view and front view of optical sensor's structure schematic are shown in Fig. 2(a) and Fig. 2(b), respectively. In Fig. 2(a) points $A_{1}, A_{2}, A_{3}$ and $A_{4}$ represent lasers' position, and $B_{1}, B_{2}, B_{3}$ and $B_{4}$ are matrix CCD cameras' position, respectively. From Fig. 2(a) one can see that matrix CCD camera should see at least quarter circumference of measurement volume in $x$ axis orientation. In Fig. $2(b), A_{i}$ and $B_{i}$ represent any one of four laser and CCD camera, respectively. Plane $A_{i} N_{1} N_{2}$ is the laser light plane, and trapezoid $V_{11} v_{22} v_{32} v_{31}$ is the field of view of CCD camera on light plane and $B_{1} O_{1}$ is the optical axis of camera.

If the half diagonal length $L L$ of square $A_{1} A_{2} A_{3} A_{4}$ is known, we can calculate the laser's horizontal angle $\varphi$ that is indicated in Fig. 2(b) easily

$$
\varphi \geq 2 \arctan (R / L L)
$$

The distance $\mathrm{H}$ and included angle $\alpha$ as shown in Fig. 2(b) must be designed elaborately. In Fig. 2(b) camera's field of view must encircle measuring volume circle. Letting Camera's horizontal and vertical field angle be $\gamma$ and $\beta$, respectively, a set of equations and inequations can be listed based on Fig. 2(a) and (b) 


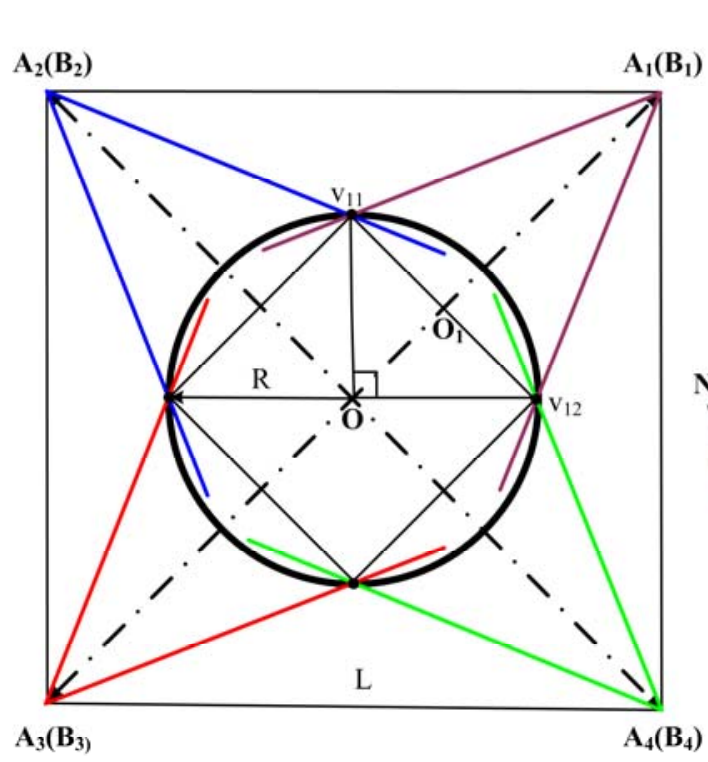

(a)

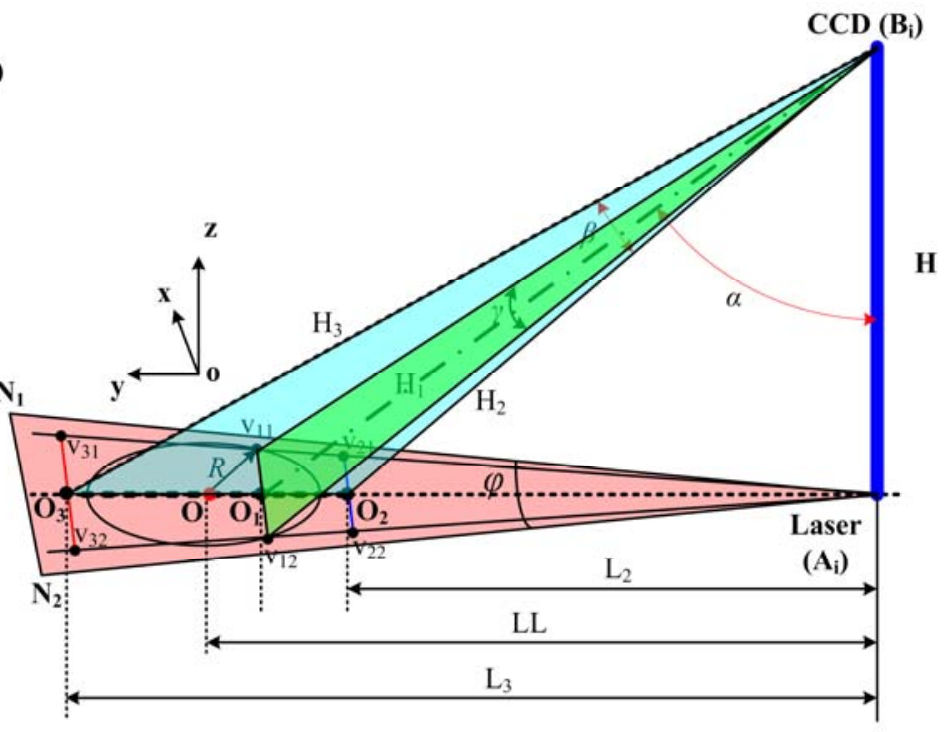

(b)

Fig. 2 Optical sensor's structure schematic.

$$
\begin{array}{r}
2 H_{1} \tan \gamma / 2=v_{11} v_{12}=\sqrt{2} R \leq r_{h} \times N_{h}^{c} \\
\left\|O_{1} O_{2}\right\|=H_{1} \sin (\beta / 2) / \sin (90+\alpha-\beta / 2) \\
\left\|O_{1} O_{3}\right\|=H_{1} \sin (\beta / 2) / \sin (90+\alpha+\beta / 2) \\
\left\|O_{1} O_{2}\right\|+\left\|O_{1} O_{3}\right\| \leq r_{v} \times N_{v}^{c}
\end{array}
$$

where $N_{h}^{c}$ and $N_{v}^{c}$ is the number of sensing units along camera horizontal and vertical direction, respectively.

Due to the categories of CCD camera combining with lens on shelf are not many, we firstly select several CCD cameras with lens and calculate parameters $H_{1}$; and then let parameter $\alpha$ change in region $\left(0,90^{\circ}\right)$ with a little step to calculate parameters $\left\|O_{1} O_{2}\right\|$ and $\left\|O_{1} O_{3}\right\|$ under the restriction inequation (5); Finally calculates the parameter $H$ and $L L$. With adequate consideration of scanner's whole sizes, the camera selected in this scanner is Adimec $1000 \mathrm{~m}$ with $8 \mathrm{~mm}$ lens, 50 frames $/ \mathrm{s}$, 1004pixels $\times 1004$ pixels. The system parameters calculated as follows: $H=392 \mathrm{~mm}, \alpha=49.5$ degrees and $L L=822 \mathrm{~mm}$. The actual typical resolutions are $0.7 \mathrm{~mm} / \mathrm{pixel}$ along $\mathrm{x}$ axis and $1.0 \mathrm{~mm} /$ pixel along $x$ axis, respectively.

The laser can be determined by $L$ and $R$. its horizontal angle $\varphi$ must be larger than 62.6 degrees.

\subsubsection{Movement mechanism module}

Optical sensor must be installed on movement mechanism module which ensures it to scan the human profile under test smoothly. The movement mechanism module designed in this system is as illustrated in Fig. 3. It is composed of a servo motor , linear guide pair and ball screw . Optical sensor is installed on slide board . In order to decrease immeasurable region, we adopt double cameras which are symmetrical to line-structure laser light source . At regular intervals along vertical direction, CCD cameras capture light stripe image series, and from those images 3D space points are calculated. 




Fig. 3 the schematic of movement mechanism.

\subsection{Motion control and image capturing module}

Optical sensing and movement mechanism module works as a whole controlled by motion control and image capturing module. Its flow chart is shown in Fig. 4. Firstly image capturing card and motion control card must be initialized. For image capturing card, we need to set parameters such as bright and contrast value; for motion control card, we need to set parameters including travel distance, acceleration and velocity, etc. If the movement begins, image capturing card captures light stripe image series and meanwhile computer records the $z$ coordinate where an image is captured. If the movement ends, the program jumps to light stripe image processing module.
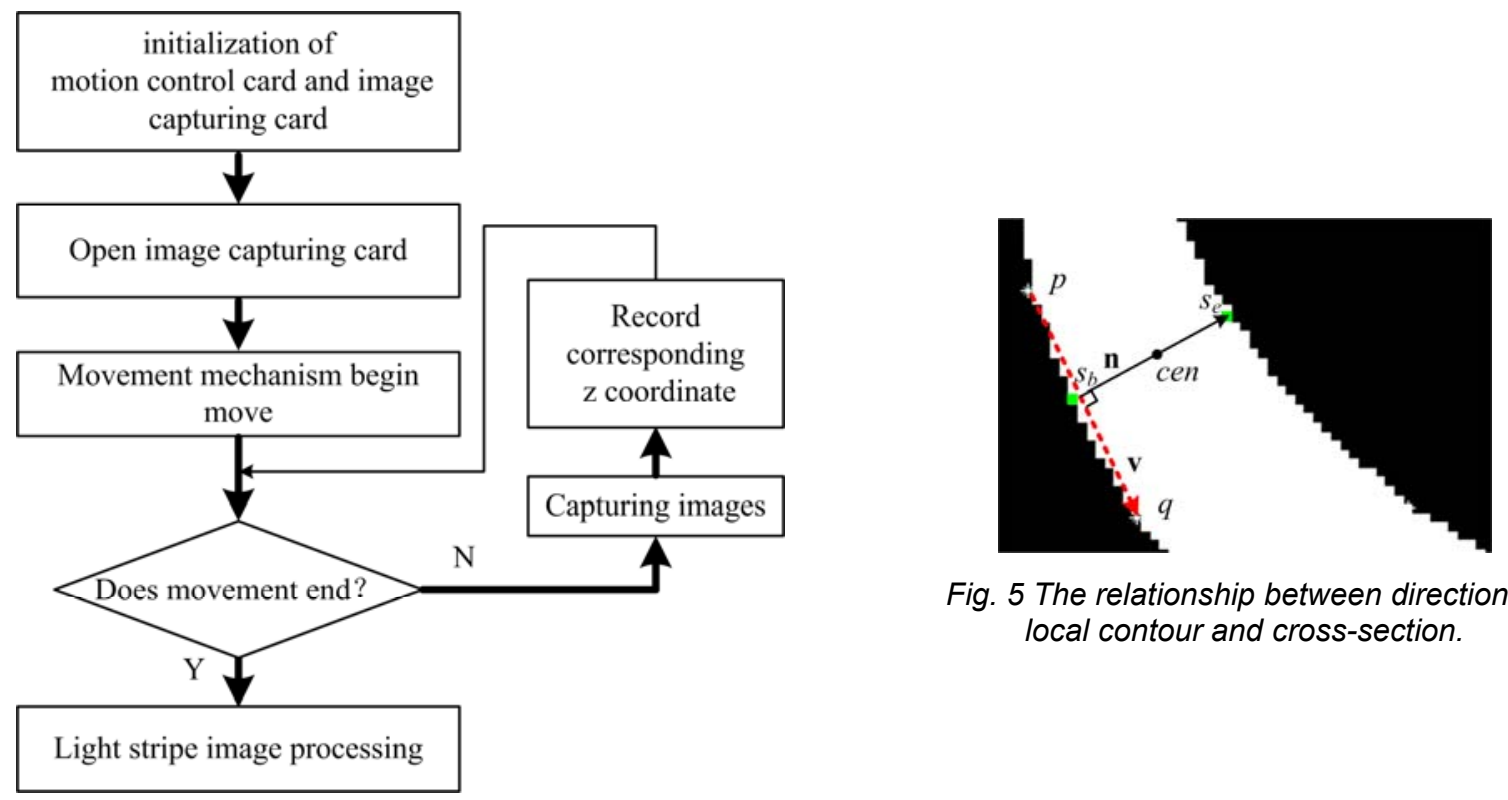

Fig. 5 The relationship between direction of local contour and cross-section.

Fig. 4 Motion control and image capturing module' flow chart.

\subsection{Light stripe image process module}

Light plane is usually generated by oblique incident laser light on the cylindrical reflector. Therefore, it has a changeable thickness. At focus position the thickness is the thinnest. it is becoming thicker and thicker far away from focus position, which results in scattering light stripe has a changeable thickness too, but 3D shape information is totally determined by its centerline, so light stripe centerline extraction is the key and basic problem in line-structured light scanning human body $3 \mathrm{D}$ scanner. In order to extract light stripe's centerline, the authors have presented a method based on contour polygon representation [15], therefore we only give the outline of this method here.

Referring to Fig. 5, the direction $\mathbf{n}$ of light stripe's cross-section is perpendicular to the direction $\mathbf{v}$ of light stripe's contour $\overline{p q}$ in a small region. So we can quickly calculate direction of local light stripe's 
cross-section through calculating the direction of local light stripe's contour.

Firstly, a set of contours $C_{i}, i=1,2 \ldots$ of light stripes are tracked in binary image using method in Ref. [16]. Secondly, using polygon $P_{i}$ represents contour $C_{i}$ satisfying that distance from any pixel on contour $C_{i}$ to polygon $P_{i}$ is less than a preliminary distance threshold $\mathrm{d}_{\mathrm{th}}$. Thirdly, for an edge $\overline{p q}$ in polygon $P_{i}$ as illustrated in Fig. 5, providing the coordinate of $p$ and $q$ is $\left(x_{p}, y_{p}\right)$ and $\left(x_{q}, y_{q}\right)$ respectively. The direction vector $\mathbf{v}_{p q}$ can be easy computed as $\left(x_{q}-x_{p}, y_{q}-y_{q}\right)$. Fourthly, due to direction $\mathbf{n}_{\text {cross }}$ of cross-section of light stripe between point $p$ and $q$ are orthogonal to $\mathbf{v}_{\overline{p q}}$, it can be calculated as $-1 / \mathbf{v}_{p q}$.

After cross-section's direction is calculated, we can calculate center point coordinate along that direction. As illustrated in Fig. 5, let $s_{b}$ be a start point, $s_{e}$ be corresponding end point, and $\mathbf{n}$ be direction vector, a scanning line $I_{\text {scan }}$ can be defined. The sub-pixel center coordinate can be computed using formula (3) and (4).

$$
\begin{aligned}
& x_{\text {cen }}=\frac{\sum_{s_{j} \in I_{\text {sean }} \& s_{j} \in \operatorname{Enner}(C)} x_{s_{j}} \times I\left(s_{j}\right)}{\sum_{s_{j} \in I_{\text {sean }} \& s_{j} \in \operatorname{Inner}(C)} I\left(s_{j}\right)} \\
& y_{\text {cen }}=\frac{\sum_{s_{j} \in l_{\text {san }} \& s_{j} \in \operatorname{Enner}(C)} y_{s_{j}} \times I\left(s_{j}\right)}{\sum_{s_{j} \in I_{\text {san }} \& s_{j} \in \operatorname{Inner}(C)} I\left(s_{j}\right)}
\end{aligned}
$$

where $s_{j}$ is on scanning line $I_{\text {scan }}, s_{j} \in \operatorname{Inner}\left(C_{i}\right)$ represents that $s_{j}$ is inside contour $C_{i}$. Using formula (3) and (4) to compute center coordinate can not only acquire sub-pixel precision, but be adaptive to the width change of cross section.

\section{System's calibration}

Based on pinhole model, the process of light stripe image capturing includes 3 transformations among 4 coordinate systems which are 3D world coordinate system $O_{w} X_{w} Y_{w} Z_{w}$, camera coordinate system $O_{c} X_{c} Y_{c} Z_{c}$, physical plane coordinate system $O_{p} X_{p} Y_{p}$ and pixel coordinate system $O_{u} X_{u} Y_{u}$, respectively. Because light plane is a horizon plane, object's $Z_{w}$ component can be thought as zero. Therefore, the transformation from $3 \mathrm{D}$ world coordinate system to pixel coordinate system can be represented by matrix

$$
s \cdot\left[\begin{array}{c}
u \\
v \\
1
\end{array}\right]=\left[\begin{array}{lll}
h_{11} & h_{12} & h_{13} \\
h_{21} & h_{22} & h_{23} \\
h_{31} & h_{32} & h_{33}
\end{array}\right] \cdot\left[\begin{array}{c}
X_{w} \\
Y_{w} \\
1
\end{array}\right]=H \cdot\left[\begin{array}{c}
X_{w} \\
Y_{w} \\
1
\end{array}\right]
$$

where $s$ is a ratio constant, $(u, v)$ represents image pixel coordinate, $\left(X_{w}, Y_{w}\right)$ represents 3D world coordinate with $Z_{w}=0 . H$ is a $3 \times 3$ homographic matrix with 8 relative independent elements. System calibration is to determine the homographic matrix $H$ for each optical sensor.

We adopt traditional method to calibrate optical sensor system. In order to provide enough calibration point pairs with high precision, we use two electric translation stages assembled orthogonally and rectangular ceramic gauge block to form calibration target as shown in Fig. 6(a). Orthogonal translation stages can drive ceramic gauge to move along arbitrary curve in $2 \mathrm{D}$ space, and offer $2 \mathrm{D}$ world coordinate with micrometer level precision. Ceramic gauge block has good optical characteristics and its four upright edges direct at 4 pillars, respectively. CCD cameras capture the light stripe scattering from two adjacent facets at any position as shown in Fig. 6(b). 


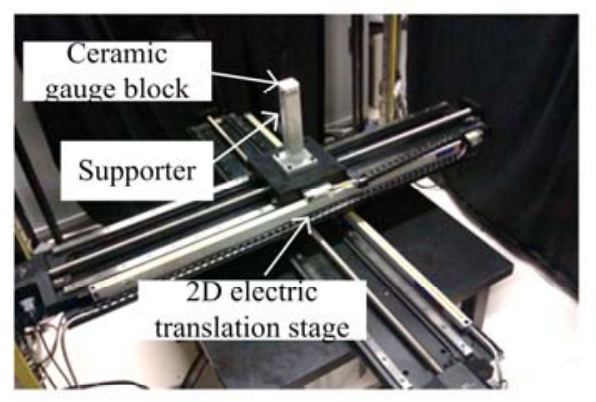

(a)

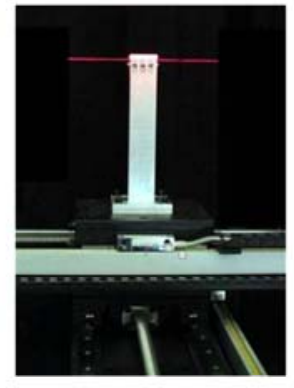

(b)



(c)

Fig. 6 Photo of calibration target and schematic of V-shape light stripe.

Calibration point's pixel coordinates are calculated as follows. Referring to Fig. 6(c), firstly the centerline of light stripe is extracted using method proposed in section 2.3 , and then it is divided into 2 segments based on its v-shape trait and each segment is fitted as straight line based on least squares criterion. Coordinates of the intersection of two fitted lines can be used as calibration point's pixel coordinate.

In the process of calibration, 2D translation stages drive ceramic gauge block to move along equal-distant concentric annulus in a certain range and on each ring two adjacent points are equal-degree. After calibration point pairs are prepared well, we use METROVISIONLAB Toolbox [17] to calculate the corresponding relations. Reprojection errors demonstrate that the mean calibration accuracy is better than $0.35 \mathrm{~mm}$ for each optical sensor. Using the calibration target designed here, we not only can calibrate each optical sensor with high precision, but also can unify each calibration result to one world coordinate system.

\section{Experimental results and analysis}

Fig. 7 shows the prototype of high resolution line-structure laser human body 3D scanner. Based on above discussion, on each pillar have 2 optical sensors, so there are 8 optical sensors totally in this scanner. The total height of each pillar is $3050 \mathrm{~mm}$, and the scanning height is $2000 \mathrm{~mm}$. scanning resolution can be changeable. When it is $2 \mathrm{~mm}$, the time used for scanning from up to bottom is 20 seconds; when it is $4 \mathrm{~mm}$, the time used for scanning from up to bottom is 10 seconds.

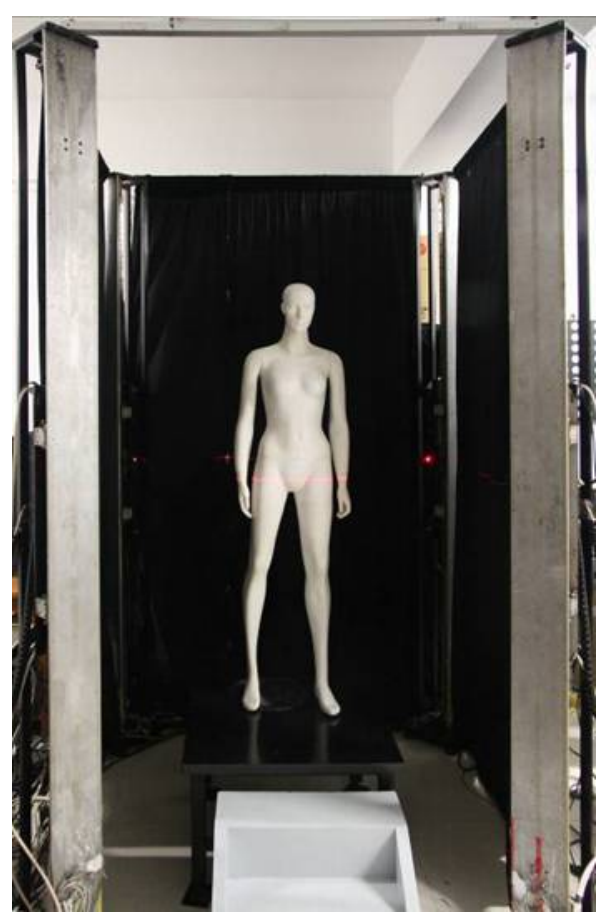

Fig. 7 The photo of high resolution line-structure laser human body $3 D$ scanner 
Fig. $8(\mathrm{a})$ and (b) show a point cloud acquired from a real person. Scanning resolution is $2 \mathrm{~mm}$, and the number of points is 1278761 . Fig. 8(c) and (d) show the surface reconstruction results of head. From surface model, one can see that some details, such as mouth, eyes and ears are very clear that indicates that the 3D human body scanner designed here has a high resolution, especially in $\mathrm{x}$ and $\mathrm{y}$ axis directions. On three positions labeled with number 1, 2 and 3 in Fig. 8(a), we cut the point cloud with horizontal plane to obtain 3 sections' point cloud that have a distance to cutting plane smaller than a predetermined distance threshold.

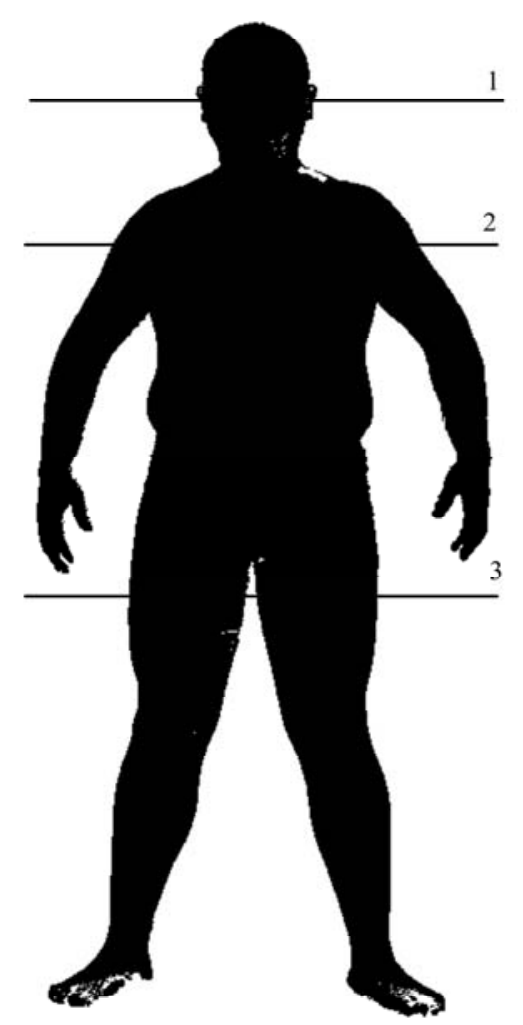

(a)

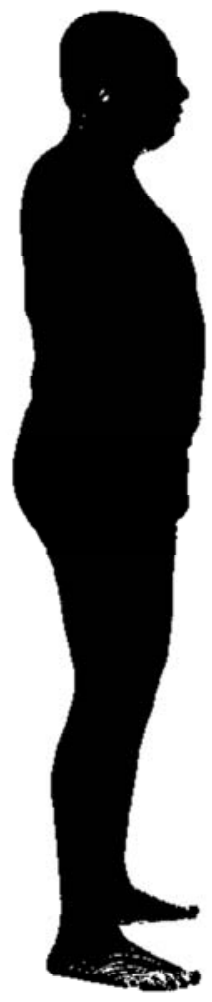

(b)

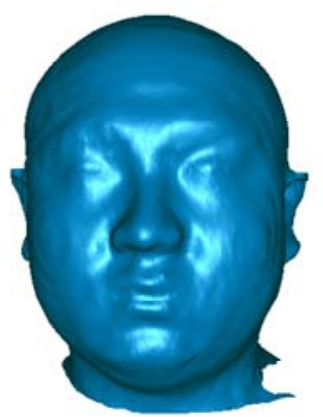

(c)

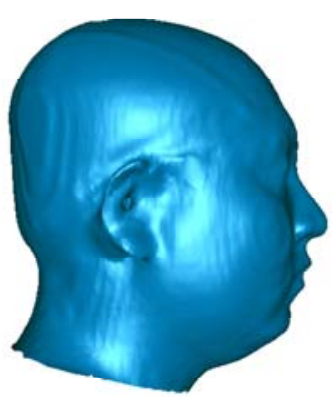

(d)

Fig. 8 3D point cloud of a real person and surface reconstruction of head.

Fig 9 show the different sections' point cloud positioned at middle of head, shoulder, and leg end. The points acquired by different optical sensors are displayed by different colors. From Fig. 9, one can see that different points by different sensors are integrated well, especially at shoulder and leg position. But at middle of head, partial adjacent point clouds have a little separation between each other. This is probably because that head position of measured human body is far away from the bottom of scanner where the calibration is pursued. Due to assembly error, the pillar can not be upright absolutely. For some parts of human body, such as behind of ears, inner thigh, etc, some points are missed due to self-occlusion and fat shape. These aspects should be improved later.

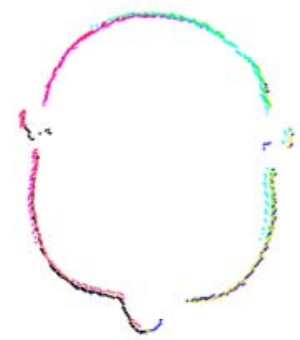

(a)

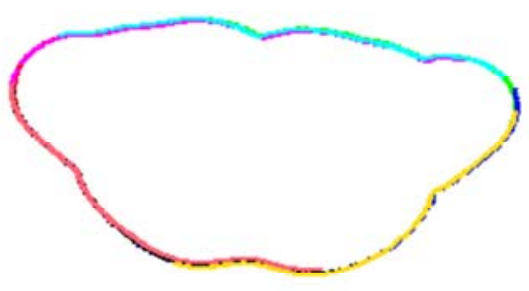

(b)

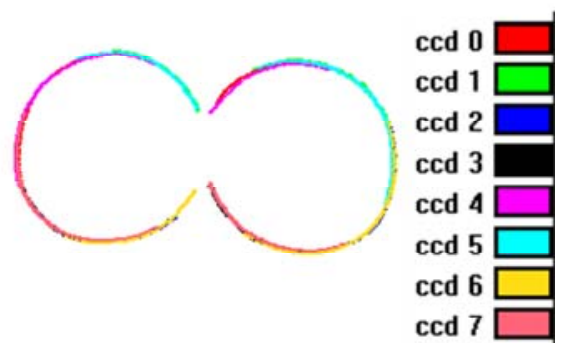

(c)

Fig. 93 section point cloud. 


\section{Conclusions}

In order to achieve accurate human body 3D digital model, this paper develops a high resolution scanner which is based on triangulation principle and line-structure laser scanning mode. Four pillars surround the measured human body, the sensors installed on each pillar move from up to down simultaneously to scan human profile. A method based on contour polygon representation is used for extracting light stripe's centerline. Dynamic calibration target is designed using 2D electric translation stages and ceramic gauge block. The reprojection mean error of optical sensor's calibration is beyond $0.35 \mathrm{~mm}$. The point cloud obtained from a real person indicates that using this scanner can acquire high resolution and precision 3D human body digital model.

\section{Acknowledgements}

The paper is supported by National natural science foundation of China (No. 61027012, No. 61177002).

\section{References}

1. Andreas V, Rainer B, Sascha H, et al. Automatic, Body Measurements Based Generation of Individual Avatars Using Highly Adjustable Linear Transformation. Digital Human Modeling, HCII2007, LNCS 4561:453-459.

2. K Lee, CAD System for Human-Centered Design, Computer-Aided Design and Computer Graphics, 2006, 3: 615-628.

3. Afzal Godil, Patrick Grother, Y Ressler. Human Identification from Body Shape. Fourth International Conference on 3D Digital Imaging and Modeling, 2003.

4. T Seitz, D Recluta, D Zimmermann, H J Wirsching. An Approach for a Human Posture Prediction Model Using Internal/External Forces and Discomfort, In: Proceedings of the SAE DHMC (Digital Human Modeling Conference) USA, 2005.

5. Colombo G, Bertetti M. Reverse engineering and rapid prototyping techniques to innovate prosthesis socket design. In: Proceeding SPIE-IS\&T Electronic Imaging, San Diego, CA, 2006, vol.6056, 60560.

6. Cherednichenko A, Assmann E, Bubb H. Computational Approach for Entry Simulation. In: Proceeding of the SAE DHMC 2006, lowa, 2006-01-2358.

7. Cappelli T, Duffy V G. Motion capture for job risk classifications incorporating dynamic aspects of work. In Proceedings of the Society of Automotive Engineers, Conference on Digital Human Modeling for Design and Engineering, 2006, SAE-DHM-2006-01-2172.

8. Uchida, N., T. Shibahara, et al. 3D Face Recognition Using Passive Stereo Vision. IEEE, 2005: 0-7803-9134-7809.

9. Lay, Y.-L., Hui-JenYang, et al. 3D face recognition by shadow moiré. Optics \& Laser Technology, 2012, 44: 148-152.

10. Yang, R., S. Cheng, et al. Flexible and accurate implementation of a binocular structured light system. Optics and Lasers in Engineering, 2008, 46: 373-379.

11. Schmalz, C., F. Forster, et al. An endoscopic 3D scanner based on structured light. Medical Image Analysis, 2012, 16: 1063-1072.

12. Gabriella Tognola, Marta Parazzini, et al. A fast and reliable system for 3D surface acquisition and reconstruction. Image and Vision Computing, 2003, 21: 295-305.

13. Michael Petrov, Andrey Talapov, et al. Optical 3D Digitizers: Bringing Life to the Virtual World. IEEE Comput.Graph. Appl, 1998, 18 (3): 28-37.

14. Park J, DeSouza G N, Kak A C. Dual-beam structured-light scanning for 3-D object modeling. Proc. of Third International Conference on 3-D Digital Imaging and Modeling, 2001, 65-72.

15. TIAN Qing-guo, GE Bao-zhen, LI Yun-peng, et al. Real-time extraction of light stripe central line based on contour polygonal representation. Optics and Precision Engineering, 2010, 18(2): 1357-1362. (In Chinese)

16. Haig, T. D., Y. Attikiouzel, et al. Border following: new definition gives improved borders. IEEE PROCEEDINGS-I, 1992, 139(2): 206-211.

17. D. Samper, J. Santolaria, J. J. Pastor, J. J. Aguilar. METROVISIONLAB Toolbox for Camera Calibration and Simulation, http://metrovisionlab.unizar.es. 